\title{
Early intervention in the management of pulmonary arterial hypertension: clinical and economic outcomes
}

This article was published in the following Dove Press journal:

ClinicoEconomics and Outcomes Research

Number of times this article has been viewed

\author{
Charles D Burger' \\ Mohamedanwar Ghandour' \\ Divya Padmanabhan Menon² \\ Haytham Helmi ${ }^{3}$ \\ Raymond L Benza ${ }^{4}$ \\ 'Division of Pulmonary, Allergy \\ and Sleep Medicine, Mayo \\ Clinic, ${ }^{2}$ Department of Internal \\ Medicine, Mayo Clinic, ${ }^{3}$ Division of \\ Transplant Medicine and Research \\ Administration, Mayo Clinic, \\ Jacksonville, FL, USA; ${ }^{4}$ Advanced \\ Heart Failure, Transplant, Mechanical \\ Circulatory Support and Pulmonary \\ Hypertension, Allegheny Health \\ Network, Pittsburg, PA, USA
}

Correspondence: Charles D Burger

Division of Pulmonary, Allergy and Sleep

Medicine, Mayo Clinic, 4500 San Pablo

Rd, Jacksonville, FL 32224, USA

Email burger.charles@mayo.edu
Abstract: Pulmonary arterial hypertension (PAH) has a high morbidity rate and is fatal if left untreated. Increasing evidence supports early intervention, possibly with initial combination therapy. PAH-specific pharmaceuticals, however, are expensive and may have serious adverse effects, particularly when used in combination. The currently dynamic health care economy reinforces the need for a review of early intervention from both outcomes and economic perspectives. We aimed to review the clinical and economic impact of PAH therapy, particularly examining drug cost, hospitalization burden, and health care economics impact, and the effect of early intervention on clinical outcomes. We searched PubMed, Scopus, Ovid, and MEDLINE databases from 2005 to 2017 for studies comparing drug cost, clinical outcomes, and hospitalization burden associated with therapy for PAH. Emerging data indicate that early therapy is effective, but drug therapy is expensive, particularly with combination therapy. Efficacy studies also generally show benefit of combination therapy for patients in World Health Organization functional class II, with a consistent decrease in hospitalization. Pharmacoeconomic studies are limited but indicate that increased pharmacy costs are at least partially offset by decreased health care utilization, particularly inpatient care. Modeling also shows a cost benefit with combination therapy at 2 years. Nonetheless, more rigorously collected health care economic data should be incorporated into future drug efficacy trials to provide a clearer understanding of the impact and the associated cost benefit of early PAH therapy. Increasing evidence in support of early intervention and combination therapy for PAH is associated with rising medication costs that are largely offset by reduced hospitalization, on the basis of the currently available literature. Nonetheless, the studies performed to date have methodologic limitations that highlight the need for prospective studies using more robust economic modeling.

Keywords: combination therapy, health care costs, hospitalization, pulmonary arterial hypertension

\section{Plain-language summary}

Pulmonary arterial hypertension $(\mathrm{PAH})$ is a progressive and debilitating disease that causes blood vessels in the lungs to thicken and narrow, which forces the heart to pump blood through the lungs at high pressures. Left untreated, the disease is relentlessly progressive, eventually causing severe strain on the heart and culminating in heart enlargement, heart failure, and death. Multiple drugs have been developed for the management of PAH over the past few decades. The medications not only help delay progression of disease but also reduce symptoms, which significantly improve quality of life. In addition, recent data suggest that early initiation of combination therapy with different classes of PAH medications provides additional benefit compared with initiation of a single agent. Extensive research has been completed and is ongoing to determine the most ideal 
medical regimens, as well as develop newer agents, yet data on the financial effects of therapy are limited. Nonetheless, some available data suggest that the cost of combination therapy may be offset by the reduction in frequent hospitalizations, because inpatient care typically represents a significant financial component of total care. The primary focus of the manuscript is to review the published literature relevant to the pharmacoeconomic aspects of PAH therapy.

\section{Introduction}

Pulmonary arterial hypertension (PAH) is a disease of vascular obstruction and destruction of the small pulmonary arteries, which results in increased pulmonary vascular resistance (PVR). ${ }^{1}$ The abnormally high PVR requires right ventricular compensation by raising the upstream pulmonary artery pressure to maintain adequate blood flow through the lungs. Ultimately, the right ventricular strain may lead to right-sided heart failure and death. As such, PAH has a high morbidity rate and is fatal if not treated. Although treatment is not universally successful, increasing evidence supports early intervention with initial combination therapy. ${ }^{2,3}$ Nonetheless, not all trials of drug combinations have yielded positive results. ${ }^{4}$ In addition, PAH-specific pharmaceuticals are expensive and may have a substantial adverse effects burden, particularly if used in combination. ${ }^{3,5-7}$

Furthermore, the increases in health care expenditures in the US present a systemic economic challenge. Health care spending in the US was $\$ 3.2$ trillion in 2015 , with prescription drug expenses estimated at $\sim 10 \%$ of all health care spending, or $\$ 325$ billion. ${ }^{8}$ Specialty pharmacy prescriptions, such as those for PAH, account for more than one-fourth of prescription drug expenses. Indeed, the growth in specialty drug spending was more than double that of traditional drug spending in 2016 (8.4\% vs 3.4\%). ${ }^{9}$ Hence, an appreciation of the clinical and economic impact of PAH therapy is important, perhaps even imperative, for patients, providers, and payers.

Currently published clinical guidelines do not adequately address these important issues. ${ }^{10,11}$ We therefore conducted a review of the relevant published literature. This article presents the results of this review, along with commentary on the current state and future direction of health care economic research in PAH.

\section{Methods}

We conducted a systematic review of the literature by searching PubMed, Scopus, Ovid, and MEDLINE databases from January 2005 to August 2017 for studies comparing drug cost, clinical outcomes, and hospitalization burden associated with therapy for PAH. The following types of studies were included: 1) prospective, placebo-controlled, randomized controlled treatment trials that included combination PAHspecific therapy; 2) systematic reviews, including meta-analyses, focused on the pharmacoeconomics of PAH-specific therapy; 3 ) studies evaluating burden of primary hospitalizations and readmissions in patients with PAH; and 4) studies examining health care models to evaluate financial impacts of single vs combination therapies for PAH. The types of patients included in the above studies were adults (age $>18$ years) with PAH and diagnostic group $1 \mathrm{PAH}$. Studies not published in English were excluded.

\section{Results}

\section{Clinical outcomes studies}

Unfortunately, PAH is a progressive disease that has no cure other than lung transplant. Pharmacologic treatments are generally lifelong and aim to improve symptoms and prolong survival. Diagnostic classification of pulmonary hypertension spans 5 separate diagnostic groups; however, drug treatment is almost exclusively limited to group 1 PAH. ${ }^{12}$ Multiple US Food and Drug Administration-approved medications indicated for group $1 \mathrm{PAH}$ are available; some of these drugs are restricted to specific World Health Organization (WHO) functional class (FC) severities. The medications target 3 major pathways: nitric oxide enhancement, endothelin-1 blockade, or prostacyclin analog or receptor agonist; therefore, the drugs may be used as monotherapy or in combination. ${ }^{11,13,14}$ Indeed, increasing evidence seems to indicate improved outcomes with combination therapy, either sequentially or as first-line treatment. . $^{3,6,7,15-18}$

Only selected efficacy studies are included in the current review (Table 1). Germane to the focus of this review on early intervention, the included studies either focused entirely on patients in FC I-II or had a substantial percentage of patients with limited symptoms. In the EARLY study (treatment of patients with mildly symptomatic PAH with bosentan [Actelion, a Janssen Pharmaceutical Company of Johnson and Johnson, New Brunswick, NJ, USA]), ${ }^{2}$ a double-blind, randomized controlled trial, patients with PAH in WHO FC II were randomly assigned to either bosentan (an endothelin receptor antagonist [ERA]) or placebo for 6 months. A combined endpoint of change in 6-minute walk distance (6MWD) and PVR was not reached; however, the PVR was decreased by $23 \%$ in the treatment group. The mean 6MWD increased from baseline by $11 \mathrm{~m}$ in the bosentan group compared with a decrease of $8 \mathrm{~m}$ in the control group, but the difference was not statistically significant. The incidence of worsening FC was lower and time to clinical worsening was delayed in the bosentan arm. On follow-up at 3.6 years, $18 \%$ of the patients had improvement in FC, which is somewhat remarkable because the study patients were in early 
Table I Representative efficacy studies of early (WHO FC I-II) intervention and relevant combination therapy studies for PAH

\begin{tabular}{|c|c|c|c|}
\hline RCT & PAH drug(s) & $\begin{array}{l}\text { Patients in } \\
\text { WHO FC I-II, \% }\end{array}$ & Outcome \\
\hline EARLY² & Bosentan & 100 & $\begin{array}{l}\text { Primary endpoint of combined 6MWD plus PVR not achieved; PVR decreased } \\
>20 \%\end{array}$ \\
\hline SERAPHIN ${ }^{\mathrm{a}, 6}$ & Macitentan & 52 & $\begin{array}{l}\text { Significant decrease (50\%) in primary endpoint of clinical events, primarily because } \\
\text { of decreased hospitalization for PAH. } 66 \% \text { of patients on background therapy }\end{array}$ \\
\hline $\mathrm{GRIPHON}^{\mathrm{a}, 7}$ & Selexipag & 47 & $\begin{array}{l}\text { Significant decrease }(40 \%) \text { in primary endpoint of clinical events, primarily because } \\
\text { of less disease progression and hospitalization. } 80 \% \text { of patients on background } \\
\text { therapy }\end{array}$ \\
\hline PATENT- $\left.\right|^{\mathrm{a}, 18}$ & Riociguat & 45 & $\begin{array}{l}\text { Significant improvement in } 6 \mathrm{MWD} \text { as primary endpoint. } 50 \% \text { of patients on } \\
\text { background therapy }\end{array}$ \\
\hline $\mathrm{AMBITION}^{3}$ & $\begin{array}{l}\text { Ambrisentan-tadalafil } \\
\text { combination }\end{array}$ & 31 & $\begin{array}{l}\text { Initial combination therapy with ambrisentan and tadalafil significantly decreased } \\
(50 \%) \text { clinical events as primary endpoint. Hospitalization in combination group } \\
\text { one-third of that with monotherapy }\end{array}$ \\
\hline COMPASS- $2^{4}$ & $\begin{array}{l}\text { Bosentan added to } \\
\text { PDE5i }\end{array}$ & 42 & $\begin{array}{l}\text { No benefit in primary endpoint of clinical events from adding bosentan to PDE5i, } \\
\text { usually sildenafil. Study lasted } 7 \text { years with } 20 \% \text { of patients missing information }\end{array}$ \\
\hline
\end{tabular}

Notes: aBackground therapy permitted.

Abbreviations: 6MWD, 6-minute walk distance; PAH, pulmonary artery hypertension; PDE5i, phosphodiesterase-5 inhibitor; PVR, pulmonary vascular resistance; RCT, randomized controlled trial; WHO FC, World Health Organization functional class.

FC II disease at the outset. The results were the first prospective randomized data to suggest that treatment earlier in the course of disease may be beneficial.

The AMBITION trial, ${ }^{3}$ a study of first-line combination therapy with ambrisentan (an ERA; Gilead Sciences, San Francisco, CA, USA) and tadalafil (a phosphodiesterase-5 inhibitor [PDE5i], thereby enhancing the nitric oxide pathway; Eli Lilly and Company, Indianapolis, IN, USA) addressed the issues of both early intervention and combination therapy. The study randomly assigned treatment-naïve patients with PAH to either initial dual therapy or monotherapy. That study design was rather novel for PAH drug studies because most prior studies used a sequential treatment approach or compared study drug with placebo and allowed pre-existing background therapy. ${ }^{3}$ The first-line combination of tadalafil and ambrisentan (titrated to maximal dose over 8 weeks) decreased the incidence of significant clinical events compared with the pooled monotherapy groups of either drug alone (hazard ratio [HR], 0.50; 95\% CI, 0.35-0.72). The effect was consistent across multiple subgroup analyses, including an examination of patients in early WHO FC II.

Of note, a recent post hoc analysis of AMBITION data by Coghlan et a ${ }^{19}$ that specifically evaluated initial combination therapy in treatment-naïve patients with connective tissue disease-associated PAH and systemic sclerosis-associated PAH showed similar findings. Patients in FC II and III who were randomly assigned to ambrisentan plus tadalafil showed risk reductions for clinical failure of $57 \%$ in connective tissue disease-associated PAH and 56\% in systemic sclerosis-associated PAH compared with monotherapy with either agent. The improvement was similar in magnitude to that seen in patients with idiopathic/hereditable PAH (49\%). In addition, secondary endpoints, including 6MWD and $\mathrm{N}$-terminal pro-brain natriuretic peptide levels, also showed improvements with combination therapy. In addition, the frequency of adverse events was not significantly different among the groups, which supports the use of initial combination therapy in this cohort as well.

Additional information regarding the benefit of combination therapy can be gleaned from studies that permitted background therapy. It is important to appreciate that such studies typically include both prevalent cases, in whom background therapy with 1 or more PAH drugs is ongoing at enrollment, and patients with new diagnoses (incident cases). Nonetheless, subgroup analysis of patients receiving background therapy who are then randomly assigned to study drug or placebo can be illustrative. Three recent randomized, placebo-controlled trials ${ }^{6,7,18}$ have demonstrated efficacy of study drug across all 3 major treatment targets, including benefit in patients already on background PAH therapy.

The SERAPHIN trial ${ }^{6}$ was a multicenter, double-blind, placebo-controlled, randomized, long-term efficacy trial that involved patients in WHO FC II, III, or IV who were treated with macitentan (an ERA; Actelion, a Janssen Pharmaceutical Company of Johnson and Johnson, New Brunswick, NJ, USA) alone or in combination with PDE5is or oral/inhaled prostanoids. Of note, there were 3 study arms: placebo, macitentan $3 \mathrm{mg}$, and macitentan $10 \mathrm{mg}$. Time to clinical worsening was significantly decreased at both the lower and higher dose of macitentan compared with placebo (more so at the 10-mg daily dose that was ultimately approved; HR, $0.55 ; P<0.001)$. Decreases in hospitalization were seen in 
both treatment arms compared with placebo but were more pronounced with the higher dose. ${ }^{20}$

Likewise, the GRIPHON study ${ }^{7}$ of selexipag (Actelion, a Janssen Pharmaceutical Company of Johnson and Johnson, New Brunswick, NJ, USA) for the treatment of PAH randomly assigned patients to selexipag (a nonprostanoid prostacyclin receptor agonist that works in the prostacyclin pathway) or placebo; however, most patients $(80 \%)$ were on background therapy. The primary endpoint of PAH-related events was significantly decreased in the selexipag group (HR, 0.6; $P<0.001$ ). Notably, a decrease in disease progression and hospitalization accounted for most of the difference.

Representing the third treatment target pathway, riociguat (Bayer Pharmaceuticals, Berlin, Germany), a soluble guanylate cyclase stimulator that increases nitric oxide, was compared with placebo in the PATENT- 1 study.$^{18}$ The primary endpoint was achieved, with an improvement in the 6MWD, as well as in secondary endpoints evaluating symptom burden, biomarkers, and hemodynamics, with a 29\% decrease in PVR. Consistent with the previous studies discussed, $50 \%$ of participants were receiving background therapy, typically an ERA.

Although adding PAH therapy in sequence generally seems to show additional benefit, it is not a uniform finding. The COMPASS- 2 trial compared bosentan with placebo in patients taking background PDE5is, usually sildenafil (Pfizer Pharmaceuticals, New York, NY, USA). ${ }^{4}$ The primary combined morbidity and mortality endpoint was not improved in the combination cohort. The study required nearly 7 years to reach sufficient clinical events to meet the predetermined power analysis. A high dropout rate was observed over the course of the study, which perhaps influenced the results. Nonetheless, the study findings were negative.

Meta-analyses provide complementary assessments of the published literature on combination therapy. One such review of 35 studies of monotherapy and combination therapy showed an overall mortality benefit of PAH treatment. ${ }^{21}$ Comparison of outcomes in the single-drug vs combination-drug studies indicated that 6MWD and hemodynamics, when available, were improved with combination therapy, but there was no difference in mortality rates. A second meta-analysis focused on available studies that examined the more modern-day, multiple-component endpoints that represent quantifiable measures of clinical worsening such as hospitalization or death. ${ }^{22}$ Analysis of 15 studies showed a significant risk reduction with combination therapy (relative risk, $0.65 ; P<0.001$ ) for clinical worsening but not death or transplant. A systematic review and network meta-analysis involving 31 randomized controlled trials with 6565 patients spanned the spectrum of currently available PAH medications. ${ }^{23}$ The frequency of clinical worsening on the study drug, alone or in combination, compared with placebo, was significantly decreased for several agents: riociguat, an ERA plus a PDE5i, and a PDE5i alone. Improvement in functional status was significant with the use of infusion prostanoids, an ERA plus a PDE5i, and an ERA alone. Only the combination of an ERA plus a PDE5i decreased the risk of hospitalization, but the result was driven by only 1 trial. Differences in mortality rate were not seen. The authors noted that the findings of the systematic review were limited by few head-to-head trials and methodologic differences.

The currently available data resulted in recommendations for risk-based assessment of disease severity to determine which arm of the treatment algorithm may be most appropriate for which patients, as outlined in the 2015 European Society of Cardiology/European Respiratory Society guidelines. ${ }^{11}$ For example, a patient with low-risk, perhaps "earlystage," disease may be characterized as WHO FC I or II and relatively preserved functional status $(6 \mathrm{MWD}>440 \mathrm{~m})$. Such patients have relatively low 1-year mortality, as validated in the REVEAL study (Registry to Evaluate Early and Longterm PAH Disease Management), with quantifiable risk scoring. ${ }^{24,25}$ Treatment considerations include monotherapy and combination therapy for WHO FC II patients, with first-line tadalafil and ambrisentan (recommendation class level 1B) based on the results of the AMBITION study. ${ }^{3}$ Nonetheless, there are existing knowledge gaps due to the lack of published trials, particularly with head-to-head comparisons of the available agents in the various drug classes. Those gaps, as well as the logistical challenges of PAH therapy in general, may contribute to the real-world experience that $95 \%$ of patients are initiated on monotherapy. ${ }^{26}$

\section{Burden of hospitalization in PAH}

Clearly, severe PAH may result in the need for hospitalization for treatment of right-sided heart failure; however, the burden of hospitalization and need for inpatient care with resulting outcome implications appears to extend beyond acute decompensation of right ventricular function. ${ }^{27}$ In the REVEAL study, ${ }^{27}$ a first hospital admission within 1 year of diagnosis predicted a high likelihood of readmission over a 3-year period. Overall, the risk of hospitalization and rehospitalization has been consistently high in published studies. ${ }^{27-29}$ Approximately 57\% of patients in a real-world database of 4009 patients required at least 1 hospitalization during follow-up. ${ }^{29}$ Indeed, $42 \%$ of those hospitalized experienced at least 1 readmission within the first year after discharge, and many had multiple readmissions. Furthermore, 
hospitalization costs $(\$ 61,922$ vs $\$ 42,455)$ and length of stay (14 vs 10 days) were greater for patients with PAH than for those with other principal diagnoses. ${ }^{29}$ Importantly, mortality rate was significantly increased in $\mathrm{PAH}$ patients with hospital admission due to a specific PAH-related presentation or symptom burden. ${ }^{27}$

As already noted, there is evidence that $\mathrm{PAH}$ treatment decreases hospitalization frequency. The AMBITION trial showed that combination therapy with ambrisentan plus tadalafil, compared with pooled monotherapy, significantly decreased clinical failure events. ${ }^{3}$ The decrease in hospitalization due to PAH was the most robust primary endpoint component (hospitalization: $4 \%$ with combined therapy vs $12 \%$ with monotherapy). Similarly, the SERAPHIN trial indicated that macitentan effectively decreased hospitalization due to PAH by $50 \%$ (HR, 0.50; 97.5\% CI, 0.34-0.75). ${ }^{6}$ Specifically, the risk of all-cause hospitalization was decreased by $19 \%$ and $32 \%$ in the 3-mg and 10-mg daily dosing arms, respectively, compared with placebo. Perhaps even more importantly, the risk of PAH-related hospitalizations was reduced by $43 \%(P<0.001)$ and $52 \%(P<0.001)$, respectively, in the 2 treatment arms. ${ }^{20}$

Decreasing the rate of hospitalizations and readmissions for patients with chronic conditions such as PAH and heart failure has emerged as a priority for physicians, policy makers, payers, and hospitals. On a broad scale, health care savings of $\$ 1$ billion or more have been estimated if preventable readmissions are avoided in the US. ${ }^{30}$ Indeed, real-world database reviews confirm the increased admission and readmission rates in patients with International Classification of Diseases, Ninth Revision diagnostic codes for pulmonary hypertension, with associated increases in the economic cost related to inpatient care. ${ }^{29}$ The potential opportunity for PAH-specific medications to modify disease course, in conjunction with favorable economic effects, is worthy of review.

\section{Average PAH drug cost}

It is important to appreciate the substantial costs of PAHspecific therapy to provide appropriate context for health care economic analysis. In the US, 12 PAH-specific medications are available across the 3 drug classes that target nitric oxide, endothelin-1, or prostacyclin pathways. The annual cost of individual agents ranges from $\$ 25,000$ to $\$ 250,000$, based on wholesale acquisition costs of published "average dosing." Of course, combination therapy would generally incur additive pharmacy costs. For example, if the provider prescribes first-line ambrisentan and tadalafil therapy on the basis of the AMBITION study, ${ }^{15}$ the total annual drug cost would be $\$ 125,802$ (\$92,804 for ambrisentan $+\$ 32,998$ for tadalafil).
Interestingly, the availability of "competitor" medications within the same class has not resulted in lower pricing. There are 3 approved ERAs in the US: bosentan, ambrisentan, and macitentan. Bosentan, the first approved ERA in the US, cost $\sim \$ 36,640$ when introduced in $2001^{31}$ and is now $\$ 110,988$ annually. The current review has not accounted for pricing negotiations, drug plan availability, patient assistance programs, or out-of-pocket expenses. Nonetheless, the expense of PAH medications has contributed to payer strategies to control the cost, such as prior authorization, tiered use, and formulary restrictions. It is critically important that drug costs are incorporated into any outcome and economic impact analysis of interventional PAH therapy, as discussed below.

\section{Clinically relevant health care economics}

Attempts to characterize and quantify the health care costs associated with PAH treatment and care delivery have often used claims-based data from health care plans, but methods are highly variable. The study cohorts may be carefully selected to be representative of true diagnostic group $1 \mathrm{PAH}$ or may represent more real-world experience. The patients may have newly diagnosed (incident) PAH or be prevalent cases. The focus of these types of analyses also varies. Total costs may be examined or the costs may be adjusted as "per patient per day," "per patient per month," or even "per member per month" (PMPM). The total health care costs may be divided by components such as, for example, pharmacy cost, outpatient visits, or inpatient stays. The effects on outcomes such as functional status, hospitalization, and survival may be included. Finally, economic modeling that incorporates pharmacy cost relative to those specific outcomes and health care utilization can provide analysis of different drugs or combinations of such. The current review will discuss some representative published literature in each of these areas (Table 2).

One analysis of absolute costs included data from January 2004 through June 2010, with 504 PAH patients selected for the study cohort. ${ }^{32}$ The estimated total health care costs actually decreased by $16 \%$ over the follow-up period of treatment ( $\$ 116,681$ at baseline vs $\$ 98,243$ at follow-up). Nonetheless, those costs for PAH were significantly higher than for principal diagnoses representative of other chronic diseases. Component costs included in the analysis were pharmacy costs, which were higher than at baseline $(\$ 38,514$ vs $\$ 6440)$. In contrast, medical costs were lower $(\$ 59,729$ vs $\$ 110,241)$, a reflection of decreased utilization. For example, average ambulatory visits and inpatient stays were decreased. Furthermore, a systematic review of the published literature between 2000 and 2014 showed direct health care costs per 
Table 2 Summary of representative health care economic publications examining the cost burden of PAH and the potential impact of $\mathrm{PAH}$-specific therapy

\begin{tabular}{|c|c|c|}
\hline Study & Data Source & Result \\
\hline Sikirica et al, $2014^{32}$ & Claims-based & Increased pharmacy costs were offset by reduced utilization, with overall decrease in total costs \\
\hline Johnson et al, $2013^{34}$ & Kaiser Permanente CO & $\begin{array}{l}\text { PPPD expenditures were increased, primarily due to pharmacy costs, regardless of newly } \\
\text { diagnosed (incident) or prevalent cases }\end{array}$ \\
\hline Burke et al, $2015^{29}$ & Claims-based & High rate of hospitalization and readmission; LOS on average increased \\
\hline Burger et al, $2014^{27}$ & REVEAL Registry & $\begin{array}{l}\text { Nearly } 60 \% \text { of patients with new diagnoses hospitalized within I year of diagnosis, mostly due } \\
\text { to PAH-related conditions }\end{array}$ \\
\hline Gu et al, $2016^{33}$ & Literature review & $\begin{array}{l}\text { Sildenafil was "a cost-effective treatment, with lower costs and better efficacy than other } \\
\text { medications" }\end{array}$ \\
\hline Oudiz et al, $2016^{35}$ & Economic model & $\begin{array}{l}\text { Initial therapy with ambrisentan plus tadalafil in symptomatic patients with PAH in WHO FC II/ } \\
\text { III would decrease the costs of hospitalization for PAH-related encounters, compared with first- } \\
\text { line monotherapy, after } 2 \text { years of therapy }\end{array}$ \\
\hline Beaudet et al, $2016^{36}$ & $\begin{array}{l}\text { Excel-based budget impact } \\
\text { model }\end{array}$ & $\begin{array}{l}\text { Initiating selexipag decreases the predictable } 2 \text {-year total cost by nearly } 15 \% \text {, corresponding to a } \\
\text { cost saving of } \$ 0.04 \text { PMPM }\end{array}$ \\
\hline
\end{tabular}

Abbreviations: CO, Colorado; LOS, length of stay; PAH, pulmonary arterial hypertension; PMPM, per member per month; PPPD, per person per day; REVEAL, Registry to Evaluate Early and Long-term PAH Disease Management; WHO FC, World Health Organization functional class.

patient per month varying from $\$ 2476$ to $\$ 11,875$ (annualized range, $\$ 29,712-\$ 142,500){ }^{33}$

Commonly, the payers are most interested in costs that are adjusted per patient or plan member. A review from Kaiser Permanente Colorado examined 3-year health care expenditures for patients with PAH. ${ }^{34}$ The analysis included 157 patients, 113 (72\%) newly diagnosed and 44 (28\%) prevalent cases. Overall, the median total per patient per day and 3-year total expenditures were $\$ 56$ and $\$ 50,599$, respectively, and were relatively equivalent for incident and prevalent cases. Interestingly, the risk of death was lower among the incident cases.

As previously discussed, patients with PAH have an increased rate of hospitalization and hospital readmissions..$^{27,28}$ The economic impact of the hospital costs is illustrated in a review of 4009 enrollees in commercial insurance and Medicare Advantage from a large US health plan, 2275 (57\%) of whom were hospitalized. ${ }^{29}$ Costs were higher for admissions for PAH than for other principal diagnoses. Specific hospitalization costs were $\sim 3$-fold higher among patients with commercial insurance than among patients covered by Medicare Advantage ( $\$ 46,118$ vs $\$ 16,319$ ). To emphasize the ongoing risk of hospitalization, among 954 patients with at least 1 readmission, $51 \%, 26 \%$, and $24 \%$ had 1,2 , and 3 or more readmissions, respectively. Interestingly, modeling can be performed using published rates of hospitalization and the decrease observed with macitentan treatment in the SERAPHIN study ${ }^{37}$; the number needed to treat with macitentan to prevent a PAH-related hospitalization was 10 patients over a 1 -year period and 4 patients over a 3 -year period.

The impact of drug treatment on health care economics has been studied using various data sources and methods.
Representative studies of both monotherapy and combination therapy will be reviewed. Patients treated with sildenafil in a large US health insurance claims database between 2005 and 2008 had a modest decrease in total health care costs, from $\$ 30,014$ to $\$ 27,605,{ }^{38}$ despite increased pharmacy costs. Emergency department visits and the percentage of patients hospitalized were notably decreased. Sildenafil generally represents the cheapest option for PAH-specific drug therapy; therefore, it commonly results in a favorable economic profile. Indeed, a systematic review of the literature determined that "sildenafil was universally reported to be a cost-effective treatment." 33

In contrast, selexipag is one of the most expensive PAHspecific pharmacotherapies. ${ }^{5}$ In the only available report of health care economic data, a model assuming 1 million plan members and a 2-year horizon calculated annual total costs to compare 2 separate scenarios: 1 with selexipag and 1 without. ${ }^{38}$ Assumptions were made regarding utilization on the basis of published literature and market research data; 2015 wholesale acquisition costs were used for drug expenses. Ultimately, the model predicted 22 patients in year 1 and 23 patients in year 2 that would be treated with drugs in the prostacyclin pathway. The expected 2 -year cost was offset by $15 \%$ with the introduction of selexipag, corresponding to a cost savings of \$0.04 PMPM.

Similarly, the health care economic impact of combination therapy requires modeling. One such model ${ }^{26}$ was developed to estimate the costs of PAH over a 3-year period in a hypothetical cohort of 100 treatment-naïve patients in WHO FC II or III to compare initial monotherapy with ambrisen$\tan$ (an endothelin antagonist) or tadalafil (a PDE5i) or the combination of both. The model parameters were based on 
the results of the AMBITION trial ${ }^{15}$ discussed previously. In year 1, PMPM costs were higher with combination therapy by $\$ 0.0244$. In year 2 , the combination therapy costs were significantly less (\$0.0059) and in year 3, a cost benefit emerged with PMPM cost (-\$0.0005). Indeed, the benefit of combination therapy over monotherapy is demonstrated by the end of year 2 (Figure 1). ${ }^{35}$

\section{Discussion}

Evidence continues to accumulate in support of early intervention for patients with PAH. Such treatment reduces clinical events, including hospitalization, a hard endpoint that may offset the expense of treatment. From a clinical perspective, the results of the AMBITION trial may change the paradigm of PAH therapy. ${ }^{3}$ Early first-line combination therapy in treatment-naïve patients clearly demonstrated a significant benefit in terms of mortality and hospitalizations for worsening PAH. The benefit was also demonstrated in patients with early disease by WHO FC II status. Most add-on treatment trials included a substantial percentage of WHO FC II patients (Table 1) and also showed benefit in patients receiving background therapy. Although sequential therapy has not been uniformly successful, most of the more recent efficacy studies support consideration of combination therapy to improve outcomes. Importantly, the benefits include avoidance of hospitalization for $\mathrm{PAH}$, an event associated with increased mortality rates. ${ }^{26}$ Because the pharmacy cost of PAH is substantial, ${ }^{5}$ an appreciation of the health care economic impact of treatment is essential to guide treatment recommendations and direct future studies.

The current review provides an assessment of current knowledge. The efficacy of approved PAH-specific therapies includes the potential for reduced health care utilization, particularly hospitalization, which may offset the increased pharmacy cost of treatment. Nonetheless, if the cost of drugs remains at the current level or increases, the challenge of demonstrating cost-benefit outcomes will remain substantial. In addition, few data are published in this regard, and there are many assumptions in health care cost modeling that may not be true in reality. Efficacy studies are under way comparing 3 drugs with 2 drugs, which may show improved clinical outcomes, but the cost will most likely be in excess of $\$ 200,000$ annually, based on wholesale acquisition costs. ${ }^{5}$ Furthermore, it does not seem likely that any newly developed drug for PAH will be lower in cost than current prices. To improve our understanding of such issues, it would be beneficial for future $\mathrm{PAH}$ research to routinely incorporate specific economic endpoints to facilitate more accurate financial modeling.

Several relevant issues were not addressed in our review, but we recognize their potential effects on any assessment of early intervention from a health care economic perspective. Insurers and payers feel responsible to control costs; therefore, multiple strategies may be used, such as formulary negotiations, tiered drug use, and restricted availability. The effect on patients is also likely to be consequential. Patients struggle not only with the symptom and psychological burden of a potentially terminal illness, but also with financial stresses that may decrease or restrict treatment options. Specific effects may include poor adherence to drug regimens to save on expense and the financial burden of the "out-of-pocket" responsibility for high-cost medications.

Hopefully, newer, more innovative approaches to treatment will provide not only clinical benefit but also more efficient use of resources. Targeted drug-delivery systems

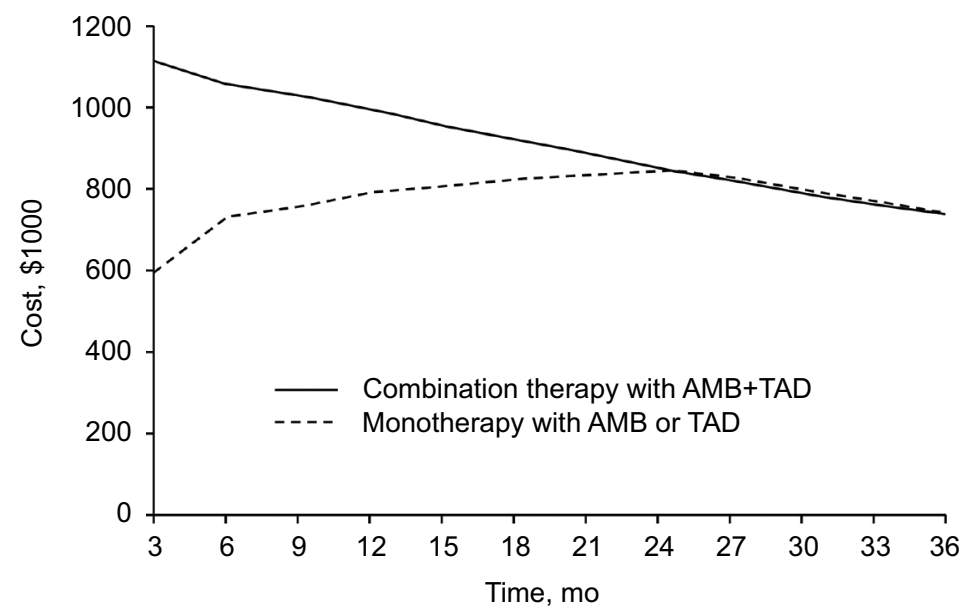

Figure I Cost-benefit model of initial therapy with AMB and TAD.

Abbreviations: AMB, ambrisentan; mo, month; TAD, tadalafil. 
using nanotechnology ${ }^{39}$ and individualized medicine involving predictive genomics ${ }^{40}$ represent such opportunities. "Implantable hemodynamic monitors (may) provide frequent hemodynamic measurements in the home environment under day-to-day conditions, allowing for a more complete evaluation of a patient's overall burden of disease."41 Such information may lead to more patient-specific medication adjustment and earlier intervention to prevent right-sided heart failure and the need for hospitalization.

To facilitate patient-centric strategies that address the challenges of health care expenses required to care for patients with rare but fatal conditions such as $\mathrm{PAH}$, research tailored to specific cost-benefit issues is required. In addition, drug pricing, health care coverage policies, and provider approaches must be adjusted with the best interests of the patient in mind.

\section{Conclusion}

Increasing evidence supports early intervention and combination therapy for PAH, but this is also associated with increasing medication costs that are largely offset by reduced hospitalization, on the basis of the currently available literature. Nonetheless, the studies performed to date have methodologic limitations that highlight the need for prospective studies using more robust economic modeling. Advances in drug delivery and patient-centric selection represent additional opportunities to enhance the cost-effectiveness of PAH treatment.

\section{Disclosure}

The authors report no conflicts of interest in this work.

\section{References}

1. Farber HW, Loscalzo J. Pulmonary arterial hypertension. NEngl J Med. 2004;351(16):1655-1665.

2. Galie N, Rubin L, Hoeper M, et al. Treatment of patients with mildly symptomatic pulmonary arterial hypertension with bosentan (EARLY study): a double-blind, randomised controlled trial. Lancet. 2008;371(9630):2093-2100.

3. Galie N, Barbera JA, Frost AE, et al. Initial Use of Ambrisentan plus Tadalafil in Pulmonary Arterial Hypertension. NEngl J Med. 2015;373(9): 834-844.

4. McLaughlin V, Channick RN, Ghofrani HA, et al. Bosentan added to sildenafil therapy in patients with pulmonary arterial hypertension. Eur Respir J. 2015;46(2):405-413.

5. Selexipag (Uptravi) for pulmonary arterial hypertension. Med Lett Drugs Ther. 2016;58(1488):21-23.

6. Pulido T, Adzerikho I, Channick RN, et al; SERAPHIN Investigators. Macitentan and morbidity and mortality in pulmonary arterial hypertension. N Engl J Med. 2013;369(9):809-818.

7. Sitbon O, Channick R, Chin KM, et al. Selexipag for the treatment of pulmonary arterial hypertension. $N$ Engl J Med. 2015;373(26): $2522-2533$
8. Centers for Medicare \& Medicaid Services. National Health Expenditure Data: Projected; 2017. Available from: https:/www.cms.gov/ Research-Statistics-Data-and-Systems/Statistics-Trends-and-Reports/ NationalHealthExpendData/NationalHealthAccountsProjected.html. Accessed August 24, 2017.

9. Express Scripts. Drug Trend Report 2016: Express Scripts; 2016. Available from: https://lab.express-scripts.com/lab/drug-trend-report. Accessed Aug 24, 2017.

10. McLaughlin VV, Archer SL, Badesch DB, et al. ACCF/AHA 2009 expert consensus document on pulmonary hypertension a report of the American College of Cardiology Foundation Task Force on Expert Consensus Documents and the American Heart Association developed in collaboration with the American College of Chest Physicians; American Thoracic Society, Inc.; and the Pulmonary Hypertension Association. J Am Coll Cardiol. 2009;53(17):1573-1619.

11. Galie N, Humbert M, Vachiery JL, et al. 2015 ESC/ERS Guidelines for the diagnosis and treatment of pulmonary hypertension: The Joint Task Force for the Diagnosis and Treatment of Pulmonary Hypertension of the European Society of Cardiology (ESC) and the European Respiratory Society (ERS): Endorsed by: Association for European Paediatric and Congenital Cardiology (AEPC), International Society for Heart and Lung Transplantation (ISHLT). Eur Heart J. 2016;37(1):67-119.

12. Simonneau G, Gatzoulis MA, Adatia I, et al. Updated clinical classification of pulmonary hypertension. J Am Coll Cardiol. 2013;62(25 Suppl):D34-D41.

13. Huisman AM, Vreugdenhil SA, van Paassen HC. Treatment of pulmonary arterial hypertension. $N$ Engl J Med. 2005;352(3):308-309; author reply 308-309.

14. Enderby CY, Burger C. Medical treatment update on pulmonary arterial hypertension. Ther Adv Chronic Dis. 2015;6(5):264-272.

15. Hoeper MM, McLaughlin VV, Barbera JA, et al. Initial combination therapy with ambrisentan and tadalafil and mortality in patients with pulmonary arterial hypertension: a secondary analysis of the results from the randomised, controlled AMBITION study. Lancet Respir Med. 2016;4(11):894-901.

16. Galie N, Jansa P, Pulido T, et al. SERAPHIN haemodynamic substudy: the effect of the dual endothelin receptor antagonist macitentan on haemodynamic parameters and NT-proBNP levels and their association with disease progression in patients with pulmonary arterial hypertension. Eur Heart J. 2017;38(15):1147-1155.

17. Bruderer S, Hurst N, Remenova T, Dingemanse J. Clinical pharmacology, efficacy, and safety of selexipag for the treatment of pulmonary arterial hypertension. Expert Opin Drug Saf. 2017;16(6):743-751.

18. Ghofrani HA, Galie N, Grimminger F, et al. Riociguat for the treatment of pulmonary arterial hypertension. N Engl J Med. 2013;369(4):330-340.

19. Coghlan JG, Galie N, Barbera JA, et al. Initial combination therapy with ambrisentan and tadalafil in connective tissue disease-associated pulmonary arterial hypertension (CTD-PAH): subgroup analysis from the AMBITION trial. Ann Rheum Dis. 2017;76(7):1219-1227.

20. Channick RN, Delcroix M, Ghofrani HA, et al. Effect of macitentan on hospitalizations: results from the SERAPHIN trial. JACC Heart Fail. 2015;3(1):1-8.

21. Liu HL, Chen XY, Li JR, et al. Efficacy and safety of pulmonary arterial hypertension-specific therapy in pulmonary arterial hypertension: a meta-analysis of randomized controlled trials. Chest. 2016;150(2):353-366.

22. Lajoie AC, Lauziere G, Lega JC, et al. Combination therapy versus monotherapy for pulmonary arterial hypertension: a meta-analysis. Lancet Respir Med. 2016;4(4):291-305.

23. Jain S, Khera R, Girotra S, et al. Comparative effectiveness of pharmacologic interventions for pulmonary arterial hypertension: a systematic review and network meta-analysis. Chest. 2017;151(1):90-105.

24. Benza RL, Miller DP, Gomberg-Maitland M, et al. Predicting survival in pulmonary arterial hypertension: insights from the Registry to Evaluate Early and Long-Term Pulmonary Arterial Hypertension Disease Management (REVEAL). Circulation. 2010;122(2):164-172. 
25. Benza RL, Gomberg-Maitland M, Miller DP, et al. The REVEAL Registry risk score calculator in patients newly diagnosed with pulmonary arterial hypertension. Chest. 2012;141(2):354-362.

26. Burger CD, Ozbay B, Riehle E, Lenhart G, White RJ. Treatment Patterns After Initial Therapy Among Pulmonary Arterial Hypertension Patients In The United States [Abstract]. Am J Respir Crit Care Med. 2016;193:A3987.

27. Burger CD, Long PK, Shah MR, et al. Characterization of first-time hospitalizations in patients with newly diagnosed pulmonary arterial hypertension in the REVEAL registry. Chest. 2014;146(5):1263-1273.

28. Frantz RP, Schilz RJ, Chakinala MM, et al. Hospitalization and survival in patients using epoprostenol for injection in the PROSPECT observational study. Chest. 2015;147(2):484-494.

29. Burke JP, Hunsche E, Regulier E, Nagao M, Buzinec P, Drake Iii W. Characterizing pulmonary hypertension-related hospitalization costs among Medicare Advantage or commercially insured patients with pulmonary arterial hypertension: a retrospective database study. $\mathrm{Am} \mathrm{J}$ Manag Care. 2015;21(3 Suppl):s47-s58.

30. Medicare Payment Advisory Commission USDOH, Human S. Medicare Payment Advisory Commission report to the Congress, March 2010. J Pain Palliat Care Pharmacother. 2010;24(3):302-305.

31. Popa A. Bosentan (Tracleer), a New Agent for the Treatment of Pulmonary Arterial Hypertension. Pharmacotherapy Update. 2002;V(2).

32. Sikirica M, Iorga SR, Bancroft T, Potash J. The economic burden of pulmonary arterial hypertension $(\mathrm{PAH})$ in the US on payers and patients. BMC Health Serv Res. 2014;14:676.
33. Gu S, Hu H, Dong H. Systematic Review of the economic burden of pulmonary arterial hypertension. Pharmacoeconomics. 2016;34(6):533-550.

34. Johnson S, Delate T, Boka A, Shaw P, Zager C. Characterizing the financial burden of pulmonary arterial hypertension within an integrated healthcare delivery system. J Med Econ. 2013;16(12):1414-1422.

35. Oudiz RJ, Mathai S, White R, Jacobsen M, Weycker D, Burger C. Cost consequences of initial combination treatment of pah with ambrisentan and tadalafil. Am J Respir Crit Care Med. 2016;193:A2953.

36. Beaudet A, Tsang Y, Hunsche E, Drake W, Bilir SP. Budget impact model for selexipag: taking a US payer perspective. Value Health. 2016;19(3):A243-A244.

37. Li H, Highland K, Cole M, et al. Minimizing burden of disease-related hospitalization among pulmonary arterial hypertension patients [Abstract]. J Manag Care Spec Pharm. 2016;22(10-a Suppl):S57.

38. Berger A, Edelsberg J, Teal S, Mychaskiw MA, Oster G. Changes in healthcare utilization and costs associated with sildenafil therapy for pulmonary arterial hypertension: a retrospective cohort study. $B M C$ Pulm Med. 2012;12:75.

39. Vaidya B, Gupta V. Novel therapeutic approaches for pulmonary arterial hypertension: Unique molecular targets to site-specific drug delivery. J Control Release. 2015;211:118-133.

40. Geraci MW, Bull TM, Tuder RM. Genomics of pulmonary arterial hypertension: implications for therapy. Heart Fail Clin. 2010;6(1):101-114

41. Kjellstrom B, Frantz RP, Benza RL, Bennett T, Bourge RC, McGoon MD. Hemodynamic ranges during daily activities and exercise testing in patients with pulmonary arterial hypertension. J Card Fail. 2014;20(7):485-491.
ClinicoEconomics and Outcomes Research

\section{Publish your work in this journal}

ClinicoEconomics and Outcomes Research is an international, peerreviewed open-access journal focusing on health technology assessment, pharmacoeconomics and outcomes research in the areas of diagnosis, medical devices, and clinical, surgical and pharmacological intervention. The economic impact of health policy and health systems

\section{Dovepress}

organization also constitute important areas of coverage. The manuscript management system is completely online and includes a very quick and fair peer-review system, which is all easy to use. Visit http://www.dovepress.com/testimonials.php to read real quotes from published authors.

Submit your manuscript here: https://www.dovepress.com/clinicoeconomics-and-outcomes-research-journal 